\title{
Effective interactions in medium heavy nuclei
}

\author{
T. Engeland, M. Hjorth-Jensen and E. Osnes \\ Department of Physics, University of Oslo, N-0316 Oslo, Norway
}

\begin{abstract}
We present a brief overview of microscopic nuclear structure approaches to nuclei with $A \sim 100-132$. The emphasis is on the shell model and theories for deriving effective interactions starting from the free interactions between nucleons. New results for ${ }^{105,106,107} \mathrm{Sb}$ are presented.
\end{abstract}

Key words: Shell model; Effective interactions

\section{Introduction}

Nuclei far from the line of $\beta$-stability are at present in focus of the nuclear structure physics community. Considerable attention is being devoted to the experimental and theoretical study of nuclei near ${ }^{100} \mathrm{Sn}$, from studies of the chain of Sn isotopes up to ${ }^{132} \mathrm{Sn}$ to e.g., nuclei near the proton drip line like ${ }^{105,106} \mathrm{Sb}$. Nuclei like ${ }^{105} \mathrm{Sb}$ and ${ }^{109} \mathrm{I}$ have recently been established as groundstate proton emitters $[1,2]$. The next to drip line nucleus for the antimony isotopes, ${ }^{106} \mathrm{Sb}$ with a proton separation energy of $\sim 400 \mathrm{keV}$, was studied recently in two experiments and a level scheme for the yrast states was proposed in Ref. [3]. Similarly, detailed spectroscopy of ${ }^{107} \mathrm{Sb}$ has also been presented recently [4].

In this contribution we focus on selected aspects of tin isotopes, with an emphasis on the connection to the underlying nucleon-nucleon interaction and large-scale shell-model studies with effective interactions. The derivation of a shell-model effective interaction is briefly discussed in section 2. Since the effective interactions employed in shell-model calculations are always the outcome of some truncations in the many-body expansion, the shell model may then provide a useful testing ground for the various approximations made. Furthermore, the shell-model wave function can be used to extract information on specific correlations in nuclei, such as pairing correlations. This is discussed 
in section 3, while section 4 contains new results from studies of Sb isotopes. Concluding remarks are presented in section 5.

\section{Effective interactions and the shell model}

Our scheme to obtain an effective two-body interaction for shell-model studies starts with a free nucleon-nucleon interaction $V$ which is appropriate for nuclear physics at low and intermediate energies. In this work we will thus choose to work with the charge-dependent version of the Bonn potential models, see Ref. [6]. The next step in our many-body scheme is to handle the fact that the repulsive core of the nucleon-nucleon potential $V$ is unsuitable for perturbative approaches. This problem is overcome by introducing the reaction matrix $G$, which in a diagrammatic language represents the sum over all ladder type of diagrams. This sum is meant to renormalize the repulsive shortrange part of the interaction. The physical interpretation is that the particles must interact with each other an infinite number of times in order to produce a finite interaction. We calculate $G$ using the double-partioning scheme discussed in e.g., Ref. [5]. Since the $G$-matrix represents just the summation to all orders of particle-particle ladder diagrams, there are obviously other terms which need to be included in an effective interaction. Long-range effects represented by core-polarization terms are also needed. In order to achieve this, the $G$-matrix elements are renormalized by the $\hat{Q}$-box method. The $\hat{Q}$-box is made up of non-folded diagrams which are irreducible and valence linked. Here we include all non-folded diagrams to third order in $G$ [5]. Based on the $\hat{Q}$-box, we compute an effective interaction $\tilde{H}$ in terms of the $\hat{Q}$-box, using the folded-diagram expansion method, see e.g., Ref. [5] for further details.

The effective two-particle interaction can in turn be used in large-scale shell model calculations. The shell model problem requires the solution of a real symmetric $n \times n$ matrix eigenvalue equation $\tilde{H}\left|\Psi_{k}\right\rangle=E_{k}\left|\Psi_{k}\right\rangle$, with $k=$ $1, \ldots, K$. At present our basic approach to finding solutions to this equation is the Lanczos algorithm; an iterative method which gives the solution of the lowest eigenstates. The technique is described in detail in Ref. [7].

In our studies the shell-model space consists of the orbits $2 s_{1 / 2}, 1 d_{5 / 2}, 1 d_{3 / 2}$, $0 g_{7 / 2}$ and $0 h_{11 / 2}$, for both protons and neutrons. For the studies of the $\mathrm{Sb}$

isotopes we used ${ }^{100} \mathrm{Sn}$ as closed shell core, while for the heavy tin isotopes we derived an effective interaction with ${ }^{132} \mathrm{Sn}$ as closed shell core, see e.g., Refs. $[3,8]$ for calculational details. The dimensionality $n$ of the eigenvalue matrix $\tilde{H}$ is increasing with increasing number of valence particles or holes. As an example, for ${ }^{116} \mathrm{Sn}$ the dimensionality of the hamiltonian matrix is of the order of $n \sim \times 10^{8}$. 


\section{Selected features of tin isotopes}

Of interest in this study is the fact that the chain of even tin isotopes from ${ }^{102} \mathrm{Sn}$ to ${ }^{130} \mathrm{Sn}$ exhibits a near constancy of the $2_{1}^{+}-0_{1}^{+}$excitation energy, a constancy which can be related to strong pairing correlations and the near degeneracy in energy of the relevant single particle orbits. As an example, we show the experimental $2_{1}^{+}-0_{1}^{+}$excitation energy from ${ }^{116} \mathrm{Sn}$ to ${ }^{130} \mathrm{Sn}$ in Table 1. Our aim is to see whether partial waves which play a crucial role in superfluidity of neutron star matter [9], viz., ${ }^{1} S_{0}$ and ${ }^{3} P_{2}$, are equally important in reproducing the near constant spacing in the chain of even tin isotopes shown in Table 1.

In order to test whether the ${ }^{1} S_{0}$ and ${ }^{3} P_{2}$ partial waves are equally important in reproducing the near constant spacing in the chain of even tin isotopes as they are for the superfluid properties of infinite neutron star matter, we study four different approximations to the shell-model effective interaction, viz.,

(1) Our best approach to the effective interaction, $V_{\text {eff }}$, contains all one-body and two-body diagrams through third order in the $G$-matrix, see Ref. [8].

(2) The effective interaction is given by the $G$-matrix only and inludes all partial waves up to $l=10$.

(3) We define an effective interaction based on a $G$-matrix which now includes only the ${ }^{1} S_{0}$ partial wave.

(4) Finally, we use an effective interaction based on a $G$-matrix which does not contain the ${ }^{1} S_{0}$ and ${ }^{3} P_{2}$ partial waves, but all other waves up to $l=10$.

In all four cases the same NN interaction is used, viz., the CD-Bonn interaction described in Ref. [6]. Table 1 lists the results. We note from this table

Table 1

$2_{1}^{+}-0_{1}^{+}$excitation energy for the even tin isotopes ${ }^{130-116} \mathrm{Sn}$ for various approaches to the effective interaction. See text for further details. Energies are given in MeV.

\begin{tabular}{lcccccccc}
\hline & ${ }^{116} \mathrm{Sn}$ & ${ }^{118} \mathrm{Sn}$ & ${ }^{120} \mathrm{Sn}$ & ${ }^{122} \mathrm{Sn}$ & ${ }^{124} \mathrm{Sn}$ & ${ }^{126} \mathrm{Sn}$ & ${ }^{128} \mathrm{Sn}$ & ${ }^{130} \mathrm{Sn}$ \\
\hline Expt & 1.29 & 1.23 & 1.17 & 1.14 & 1.13 & 1.14 & 1.17 & 1.23 \\
$V_{\text {eff }}$ & 1.17 & 1.15 & 1.14 & 1.15 & 1.14 & 1.21 & 1.28 & 1.46 \\
$G$-matrix & 1.14 & 1.12 & 1.07 & 0.99 & 0.99 & 0.98 & 0.98 & 0.97 \\
${ }^{1} S_{0} G$-matrix & 1.38 & 1.36 & 1.34 & 1.30 & 1.25 & 1.21 & 1.19 & 1.18 \\
$\mathrm{No}^{1} S_{0} \&{ }^{3} P_{2}$ in $G$ & & & & & 0.15 & -0.32 & 0.02 & -0.21 \\
\hline
\end{tabular}

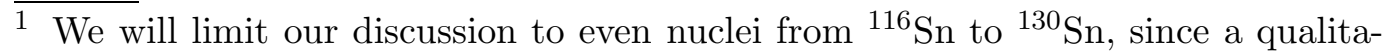
tively similar picture is obtained from ${ }^{102} \mathrm{Sn}$ to ${ }^{116} \mathrm{Sn}$. 
that the three first cases nearly produce a constant $2_{1}^{+}-0_{1}^{+}$excitation energy, with our most optimal effective interaction $V_{\text {eff }}$ being closest the experimental data. The bare $G$-matrix interaction, with no folded diagrams as well, results in a slightly more compressed spacing. This is mainly due to the omission of the core-polarization diagrams which typically render the $J=0$ matrix elements more attractive. Such diagrams are included in $V_{\text {eff }}$. Including only the ${ }^{1} S_{0}$ partial wave in the construction of the $G$-matrix (case 3), yields in turn a somewhat larger spacing. This can again be understood from the fact that a $G$-matrix constructed with this partial wave only does not receive contributions from any entirely repulsive partial wave. It should be noted that our optimal interaction, as demonstrated in Ref. [8], shows a rather good reproduction of the experimental spectra for both even and odd nuclei. Although the approximations made in cases 2 and 3 produce an almost constant $2_{1}^{+}-0_{1}^{+}$ excitation energy, they reproduce poorly the properties of odd nuclei and other excited states in the even $\mathrm{Sn}$ isotopes.

However, the fact that the first three approximations result in a such a good reproduction of the $2_{1}^{+}-0_{1}^{+}$spacing may hint to the fact that the ${ }^{1} S_{0}$ partial wave is of paramount importance. If we now turn the attention to case 4 , i.e., we omit the ${ }^{1} S_{0}$ and ${ }^{3} P_{2}$ partial waves in the construction of the $G$ matrix, the results presented in Table 1 exhibit a spectroscopic catastrophe. We do also not list eigenstates with other quantum numbers. For e.g., ${ }^{126} \mathrm{Sn}$ the ground state is no longer a $0^{+}$state, rather it carries $J=4^{+}$while for ${ }^{124} \mathrm{Sn}$ the ground state has $6^{+}$. The first $0^{+}$state for this nucleus is given at an excitation energy of $0.1 \mathrm{MeV}$ with respect to the $6^{+}$ground state. The general picture for other eigenstates is that of an extremely poor agreement with data. Since the agreement is so poor, even the qualitative reproduction of the $2_{1}^{+}-0_{1}^{+}$spacing, we defer from performing time-consuming shell-model calculations for ${ }^{116,118,120,122} \mathrm{Sn}$.

\section{Shell model studies of the proton drip line nuclei ${ }^{105,106,107} \mathrm{Sb}$}

We present recent results for ${ }^{105} \mathrm{Sb},{ }^{106} \mathrm{Sb}$ and ${ }^{107} \mathrm{Sb}$ in Table 2 . The calculations use ${ }^{100} \mathrm{Sn}$ as closed shell core with an effective interaction for the four, five or six valence neutrons and one valence proton based on the CD-Bonn nucleonnucleon interaction [6]. The experimental spin assignements for ${ }^{105} \mathrm{Sb}$ and ${ }^{106} \mathrm{Sb}$ are tentative. There are also many more theoretical states than reported in the enclosed table.

The high spin level scheme of ${ }^{105} \mathrm{Sb}$ resembles the level scheme of ${ }^{107} \mathrm{Sb}$ up to $J=19 / 2$ [4]. When compared to ${ }^{107} \mathrm{Sb}$ the ${ }^{105} \mathrm{Sb}$ level scheme shows similar trends as when going from ${ }^{106} \mathrm{Sn}$ to ${ }^{104} \mathrm{Sn}$. That means that coupling a $d_{5 / 2}$ proton to a ${ }^{104} \mathrm{Sn}$ core is appropriate to describe the observed states. The 
calculation favors $J^{\pi}=5 / 2^{+}$for the ground state in agreement with the suggestion from proton decay data. In this state the valence proton is mainly in the $d_{5 / 2}$ orbit and the two neutron pairs are almost evenly distributed over the $d_{5 / 2}$ and $g_{7 / 2}$ neutron orbits. The situation is very similar in the $9 / 2^{+}$and $13 / 2^{+}$states, while the $\nu g_{5 / 2}^{3} g_{7 / 2}^{1}$ configuration exhausts the largest parts of the wave functions of the $15 / 2^{+}$and $17 / 2^{+}$states. The neutron part of the wave function of the $19 / 2^{+}$state is almost identical to the $17 / 2^{+}$state. However, since $17 / 2^{+}$is the maximum spin for the $\pi g_{5 / 2}^{1} \nu g_{5 / 2}^{1} g_{7 / 2}^{1}$ configuration, the odd proton resides almost exclusively in the $g_{7 / 2}$ orbit in the $19 / 2^{+}$state. For proton degrees of freedom the $s_{1 / 2}, d_{3 / 2}$ and $h_{11 / 2}$ single-particle orbits give essentially negligible contributions to the wave functions and the energies of the excited states, as expected. For neutrons, although the single-particle distribution for a given state is also negligible, these orbits are important for a good describtion of the energy spectrum, as also demonstrated in large-scale shell-model calculations of tin isotopes [8]. Similar picturer applies to ${ }^{106} \mathrm{Sb}$ and ${ }^{107} \mathrm{Sb}$ as well, see Refs. [3,4]. The wave functions for the various states are to a large extent dominated by the $g_{7 / 2}$ and $d_{5 / 2}$ single-particle orbits for neutrons $(\nu)$ and the $d_{5 / 2}$ single-particle orbit for protons $(\pi)$. The $\nu g_{7 / 2}$ and $\nu d_{5 / 2}$ single-particle orbits represent in general more than $\sim 90 \%$ of the total neutron single-particle occupancy, while the $\pi d_{5 / 2}$ single-particle orbits stands for $\sim 80-90 \%$ of the proton single-particle occupancy. The other single-particle orbits play an almost negligible role in the structure of the wave functions.

Table 2

Low-lying states of ${ }^{105,106,107} \mathrm{Sb}$, theory and experiment. Energies in $\mathrm{MeV}$.

\begin{tabular}{ccc|ccc|ccc}
\hline \multicolumn{3}{c|}{${ }^{105} \mathrm{Sb}$} & \multicolumn{3}{c|}{${ }^{106} \mathrm{Sb}$} & \multicolumn{3}{c}{${ }^{107} \mathrm{Sb}$} \\
$J_{i}^{\pi}$ & Exp & Theory & $J_{i}^{\pi}$ & Exp & Theory & $J_{i}^{\pi}$ & Exp & Theory \\
\hline $5 / 2^{+}$ & 0 & 0 & $2^{+}$ & 0 & 0 & $5 / 2^{+}$ & 0 & 0 \\
$9 / 2^{+}$ & 1.22 & 1.22 & $4^{+}$ & 0.10 & 0.25 & $7 / 2^{+}$ & 0.77 & 0.69 \\
$13 / 2^{+}$ & 1.84 & 1.94 & $5^{+}$ & 0.32 & 0.54 & $9 / 2^{+}$ & 1.06 & 1.08 \\
$15 / 2^{+}$ & 2.21 & 2.10 & $6^{+}$ & 0.44 & 0.66 & $11 / 2^{+}$ & 1.79 & 1.80 \\
$17 / 2^{+}$ & 2.50 & 2.41 & $7^{+}$ & 0.89 & 1.34 & $13 / 2^{+}$ & 1.90 & 1.94 \\
$19 / 2^{+}$ & 2.99 & 2.94 & $8^{+}$ & 1.53 & 1.78 & $15 / 2^{+}$ & 2.24 & 2.38 \\
$23 / 2^{+}$ & 3.73 & 4.09 & $10^{+}$ & 2.26 & 2.57 & $17 / 2^{+}$ & 2.75 & 2.83 \\
\hline \multicolumn{1}{c}{}
\end{tabular}

\section{Conclusions}

In summary, shell-model calculations with realistic effective interactions of the newly reported low-lying yrast states of the proton drip line nuclei ${ }^{105,106,107} \mathrm{Sb}$, 
reproduce well the experimental data. Since the wave functions of the various states are to a large extent dominated by neutronic degrees of freedom and neutrons are well bound with a separation energy of $\sim 8 \mathrm{MeV}$, this may explain why a shell-model calculation, within a restricted model space for a system close to the proton drip line, gives a satisfactory agreement with the data.

For the Sn isotopes we have shown that the ${ }^{1} S_{0}$ and ${ }^{3} P_{2}$ partial waves, which are crucial for our understanding of superfluidity in neutron star matter, are equally important in order to reproduce the $2_{1}^{+}-0_{1}^{+}$excitation energy of the even $\mathrm{Sn}$ isotopes. Omitting these waves, especially the ${ }^{1} S_{0}$ wave, results in a spectrum which has essentially no correspondence with experiment.

Further analysis of nuclei such as Ag, Cd, In near $A \sim 100$ and Sn isotopes near $A \sim 132$ are in progress.

We are much indebted to Cyrus Baktash, David Dean, Hubert Grawe and Matej Lipoglavšek for many discussions on properties of nuclei near $A \sim 100$.

\section{References}

[1] R. J. Tighe, D. M. Moltz, J. C. Batchelder, T. J. Ognibene, M. W. Rowe, and J. Cerny, Phys. Rev. C49 (1994) R2871; M. Lipoglavšek et al., in preparation

[2] C. -H. Yu et al., Phys. Rev. C59 (1999) R1834.

[3] D. Sohler et al., Phys. Rev. C59 (1999) 1324; for a shell-model analysis see e.g., T. Engeland, M. Hjorth-Jensen and E. Osnes, Phys. Rev. C 61 (2000) 021302(R).

[4] D. R. LaFosse et al., Phys. Rev. C62 (2000) 014305.

[5] M. Hjorth-Jensen, T. T. S. Kuo and E. Osnes, Phys. Reports 261 (1995) 125.

[6] R. Machleidt, F. Sammarruca and Y. Song, Phys. Rev. C 53 (1996)

[7] R.R. Whitehead, A. Watt, B.J. Cole and I. Morrison, Adv. Nucl. Phys. 9 (1977) 123.

[8] A. Holt, T. Engeland, M. Hjorth-Jensen, and E. Osnes, Nucl. Phys. A634 (1998) 41.

[9] H. Heiselberg and M. Hjorth-Jensen, Phys. Rep. 328 (2000) 237. 